\title{
21
}

\section{Relation Between Parameters Sensitivities and Dimensional Invariance on Stochastic Materials Design of Fibrous Composite Laminates}

\author{
H. Nakayasu ${ }^{\mathrm{a}}$ \\ ${ }^{a}$ Department of Industrial Management, Osaka Institute of Technology \\ 5-16-1, Omiya, Asahi-ku Osaka 535, Japan \\ E-mail: nakayasu@nak101.dim.oit.ac.jp
}

This paper deals with the relation between parameters sensitivities and dimensional invariance on stochastic materials design of fibrous composite laminates. For the materials design of composite materials, there are several cases of the definition of the nominal safety factor. In this materials design, though the safety measures such as safety margins are identical with that by another definition of safety factor in deterministic design, it is not always verified that these consistencies are kept in stochastic fields because of the differences of the numbers of basic random variables. Therefore it is important problem in stochastic materials design whether there is consistency for any definition of safety factor or not.

\section{INTRODUCTION}

In the materials design of composite materials, there are several cases of the definition of safety factor in order to gain the margin of safety design for strength and modulus[1]. Each definition is essentially derived because of the intrinsic design code or calibration to practical specification. However, though the safety measure such as safety margin is coincided with that by another definition of safety factor in deterministic design, it is not always verified that these consistencies keep in stochastic fields because of the discrepancy of the number of random variables. This design problem arises from the dimensional invariability of the model in stochastic field[2],[3].

As the actual material design of composite laminates has many parameters with uncertainty such as load, strength, modulus, dimensions, and so on, all of these parameters must be evaluated in the probabilistic fields. However it has not been verified that the safety margin obtained by the nominal safety factor under the specified strain conditions in the deterministic analysis coincides with those under the strain conditions in the probabilistic field.

In this paper it is discussed that the differences of the definition of safety factor with different random parameters yield how sensitive to the final measure of reliability analysis. For the purpose of the practical stochastic materials design of composite materials, the sensitivity study on reliability of uni-directional fiber reinforced composite laminates are performed based on the present first order reliability method(FORM)[4]. Two kinds of sensitivity measures such as physical and stochastic sensitivity are defined and the relation between these sensitivities.

\section{LIMIT STATE AND SENSITIVITIES OF COMPOSITE LAMINATE}

\subsection{Limit state function by quadratic polynomial failure criteria}

When the coordinate system of unidirectional composite laminate is defined as Figure 1 


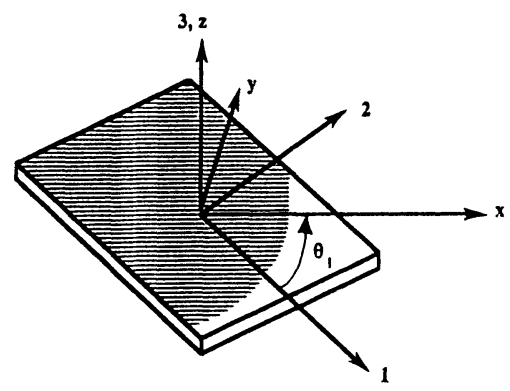

Figure 1. Coordinates system and relation stress and strain components of unidirectional composites laminates.

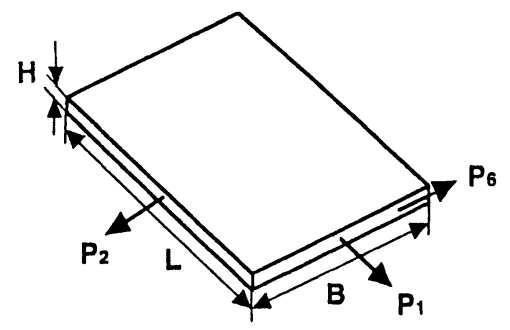

Figure2. Loads and dimensional between parameters.

and applied loads and dimensional parameters are shown in Figure 2, the typical failure criteria for materials design by Tsai-Wu[5], which is based on the mechanical behavior of composite laminates, are formulated as follows:

(1) On stress space

$g(X)=1-\sigma_{\mathbf{x}}^{\mathrm{T}} \mathbf{F}_{\mathrm{A}, \mathbf{x}} \boldsymbol{\sigma}_{\mathbf{x}}-\mathbf{F}_{\mathrm{B}, \mathrm{x}}^{\mathrm{T}}$

where $\mathbf{F}_{\mathrm{A}, \mathrm{x}}$ and $\mathbf{F}_{\mathrm{B}, \mathrm{x}}$ are strength parameter matrix and vector as

$\mathbf{F}_{\mathrm{A}, \mathrm{x}}=\left[\begin{array}{ccc}F_{\mathrm{xx}} & F_{\mathrm{xy}} & 0 \\ & F_{y y} & 0 \\ \text { sym. } & & F_{s s}\end{array}\right], \quad \mathbf{F}_{\mathrm{B}, \mathrm{x}}=\left\{\mathrm{F}_{\mathrm{x}} \mathrm{F}_{\mathrm{y}} 0\right\}^{\mathrm{T}}$

$F_{x x}=\left(\sigma_{L, t} \cdot \sigma_{L, c}\right)^{-1}, F_{y y}=\left(\sigma_{T, z} \cdot \sigma_{T, c}\right)^{-1}, F_{x y}=k_{12}\left(F_{x x} \cdot F_{y y}\right)^{1 / 2}$,

$F_{s s}=\left(\sigma_{L T}\right)^{-2}, F_{x}=\left(\sigma_{L, t}\right)^{-1}-\left(\sigma_{L, c}\right)^{-1}, F_{y}=\left(\sigma_{T, t}\right)^{-1}-\left(\sigma_{T, c}\right)^{-1}$

$\sigma_{\mathrm{L}, \mathrm{t}}$ :tensile strength on fibrous direction $\mathrm{x}$

$\sigma_{L, c}:$ compressive strength on fibrous direction $x$

$\sigma_{\mathrm{T}, t}:$ tensile strength on transverse direction $y$

$\sigma_{\mathrm{T}, \mathrm{c}}$ :compressive strength on transverse direction $\mathrm{y}$

$\sigma_{\text {LT }}$ :shear strength on $\mathrm{x}-\mathrm{y}$

$\varepsilon_{L, t}$ :ultimate tensile strain on fibrous direction $\mathrm{x}$

$\varepsilon_{\mathrm{L}, \mathrm{c}}$ :ultimate compressive strain on fibrous direction $\mathbf{x}$

$\varepsilon_{\mathrm{T}, \mathrm{t}}$ :ultimate tensile strain on transverse direction $\mathrm{y}$

$\varepsilon_{\mathrm{T}, \mathrm{c}}$;ultimate compressive strain on transverse direction y

From the definitions of limit state function of $\mathrm{i}$-th element, the state of $\mathrm{i}$-th element is divided into three categories:

$\mathrm{V}=\left\{\mathrm{g}_{\mathrm{i}}(\mathbf{X}) \leq 0\right\}, \mathrm{S}=\left\{\mathrm{g}_{\mathrm{i}}(\mathbf{X})>0\right\}, \mathrm{G}=\left\{\mathrm{gi}_{\mathrm{i}}(\mathbf{X})=0\right\}$

$\mathrm{V}, \mathrm{S}$, and $\mathrm{G}$ expresses the failure domain, safety domain, and failure surface, respectively. 
(2) On strain space

$g(X)=1-\varepsilon_{\mathbf{x}}^{T} G_{A, \mathbf{x}} \varepsilon_{\mathbf{x}}-G_{B, \mathbf{x}}^{T} \varepsilon_{\mathbf{x}}$

where $\mathbf{G}_{\mathrm{A}, \mathbf{x}}$ and $\mathbf{G}_{\mathrm{B}, \mathrm{x}}$ are strength parameter matrix and vector which are obtained by

$$
\mathbf{G}_{\mathrm{A}, \mathbf{x}}=\mathbf{Q}_{\mathrm{x}}^{\mathrm{T}} \mathbf{F}_{\mathrm{A}, \mathbf{x}} \mathbf{Q}_{\mathbf{x}}
$$

$$
\mathbf{G}_{\mathrm{B}, \mathrm{x}}^{\mathrm{T}}=\mathbf{F}_{\mathrm{B}, \mathrm{x}}^{\mathrm{T}} \mathbf{Q}_{\mathbf{x}}
$$

\subsection{Measures of sensitivity to random parameters}

If the failure domain $\mathrm{V}$ is rewritten by

$V=\left\{\mathbf{u}: \alpha^{T} \mathbf{u}+\beta \leq 0\right\}$

the safety index which is a common measure of structural safety instead of safety factor is obtained by the relations.

$P_{f}=P[\mathrm{U} \in V]=\Phi(-\beta)$

In Eq.(8), standardized normal random vector has mean and standard deviation vectors

$\mathbf{m}=\left\{\mathrm{m}_{1}, \mathrm{~m}_{2}, \mathrm{~m}_{3}, \ldots, \mathrm{m}_{\mathrm{n}}\right\}^{\mathrm{T}}=\left\{\begin{array}{lllll}0 & 0 & 0 & \ldots & 0\end{array}\right\}^{\mathrm{T}}=\mathbf{0}$,

$\sigma=\left\{\sigma_{1}, \sigma_{2}, \sigma_{3}, \ldots, \sigma_{n}\right\}^{T}=\left\{\begin{array}{llll}1 & 1 & 1 & \ldots 1\end{array}\right\}^{\mathrm{T}}=1$,

respectively.

Now the equivalent safety index $\beta_{\mathrm{E}}$ should be defined as well as Eq.(8)

$\beta_{E}=-\Phi^{-1}(P[\mathrm{U} \in V])=-\Phi^{-1}\left(P_{f}\right)$

which is corresponding to $P_{f}$ equivalently, where $\Phi^{-1}($.$) indicates the inverse function of$ cumulative function of standard normal distribution function.

In order to evaluate the sensitivity of $X_{i}$ to $\beta_{E}$, it is considered that the derivatives of $\beta_{E}$ on $\mathrm{m}_{\mathrm{i}}$ and $\sigma_{\mathrm{i}}$ are useful. Therefore, two kinds of measure of sensitivity

$s_{1}=\left.\frac{\partial \beta_{E}}{\partial m_{i}}\right|_{\substack{n \rightarrow 0 \\ \sigma \rightarrow 1}}$

$$
s_{2}=\left.\frac{\partial \beta_{E}}{\partial \sigma_{i}}\right|_{\substack{m \rightarrow 0 \\ \sigma \rightarrow 1}}
$$

should be derived from the relation 


$$
\begin{aligned}
& \beta_{E}=-\Phi^{-1}\left(P_{f}\right)=-\Phi^{-1}\left(P\left[\alpha^{T} \mathrm{u} \leq-\beta\right]\right)=-\Phi^{-1}\left(P\left[\sum_{i=1}^{n} \alpha_{i}\left(U_{i} \sigma_{i}+m_{i}\right)+\beta \leq 0\right]\right) \\
& =-\Phi^{-1}\left(P\left[\sum_{i=1}^{n} \alpha_{i} \sigma_{i} U_{i} \leq-\beta+\sum_{i=1}^{n} \alpha m_{i}\right]\right)
\end{aligned}
$$

which can be expressed by the Hesse Form

$$
\beta_{E}=-\frac{-\beta+\sum_{i=1}^{n} \alpha_{i} m_{i}}{\left(\sum_{i=1}^{n}\left(\alpha_{i} \sigma_{i}\right)^{2}\right)^{1 / 2}} \text {. }
$$

Thus the measure of sensitivity are finally expressed by the safety index and gradients of failure surface on transformed U-space.

$$
s_{1}=\left.\frac{\partial \beta_{E}}{\partial m_{i}}\right|_{\substack{n \rightarrow 0 \\ \sigma \rightarrow 1}}-\alpha_{i}
$$

$$
s_{2}=\left.\frac{\partial \beta_{E}}{\partial \sigma_{i}}\right|_{\substack{m \rightarrow 0 \\ \sigma \rightarrow 1}}=-\beta \alpha_{i}^{2}
$$

The $s_{1}$ and $s_{2}$ mean the perturbation of $\beta_{E}$ on $m_{i}$ and $\sigma_{i}$. The former is a sensitivity on mean of $\mathrm{X}_{\mathrm{i}}$ which is called physical sensitivity. On the other hand, the latter is a sensitivity on standard deviation of $\mathrm{X}_{\mathrm{i}}$ which is called stochastic sensitivity.

\section{NUMERICAL ANALYSIS}

\subsection{Definition of the three kinds of safety factor}

Consider the load condition of a unidirectional carbon/epoxy laminate (T300/5208) as shown in Fig. 2 with three kinds of the definitions of safety factor for materials design as:

1) Definition of safety factor on ultimate strength (Type I)

$$
\begin{aligned}
& S F 1_{1}=\sigma_{L, t} / \sigma_{1}^{*}\left(\sigma_{1}^{*} \geq 0\right) \text { or } \sigma_{L, c} / \sigma_{1}^{*}\left(\sigma_{1}^{*}<0\right) \\
& S F 1_{2}=\sigma_{T, t} / \sigma_{2}^{*}\left(\sigma_{2}^{*} \geq 0\right) \text { or } \sigma_{T, c} / \sigma_{2}^{*}\left(\sigma_{2}^{*}<0\right) \\
& S F 1_{3}=\sigma_{s} / \sigma_{6}^{+}
\end{aligned}
$$

2) Definition of safety factor on in-plane load (Type II)

$$
S F 2_{1}=P_{1} / P_{1}^{*}, S F 2_{2}=P_{2} / P_{2}^{*}, S F 2_{3}-P_{6} / P_{6}^{*}
$$

3) Definition of safety factor on dimensional factor (Type III)

$$
S F 3_{1}=B^{*} / B, S F 3_{2}=L^{*} / L, S F 3_{3}=H^{*} / H
$$


In the above equations, $(.)^{*}$ means the nominal value of each factor in every definition of safety factor. Since the load condition subjected to composite laminate is shown in Fig. 2, the mean stress on principle axis will be calculated from the basic relation

$$
\sigma_{1}=\frac{P_{1}}{B H}, \sigma_{2}=\frac{P_{2}}{L H}, \sigma_{6}=\frac{P_{6}}{B H}
$$

which is in correspondence to these definition of safety factor. These mean stresses by Eq.(21) is transformed into in-plane strain through stiffness matrix or compliance matrix which is composed of modulus factors. In the stochastic materials designs of composite materials, the parameters such as strength factors, modulus factors, load factors, lamination factors, and dimensional factors must be taken into account of as random variables.

Many research works on reliability based materials design of composite materials usually start from the Type I definition of nominal safety factor. Unfortunately there are differences in the number of random variables as shown in Table 1 which is dependent on the types of the definition of safety factor. Because the limit state function which is dependent on the selective failure law on composite laminates requires several kind of random parameters whose number is shown in Table 1. If the value of the safety index does not coincide with that of another definition, the designer must be modify the value of the design variables in practical design to keep equivalent safety margin according to the selection of the definition of safety factor. This design problem arises from the lack of dimensional invariability. Therefore the degree of the differences of the value of safety index should be evaluated for Eqs.(18)-(20) and the relation to the sensitivity of random parameter should be also discussed.

\subsection{Evaluation results and some considerations}

Numerical analysis of the graphite epoxy unidirectional composite laminates subjected to off axis loads was examined for the verification of the existence of the lack of dimensional invariability and the consideration of the relation to the sensitivity. Table 2 shows the values of

Table 1 Number of random variables corresponding to the definition of safety factor.

\begin{tabular}{|c|c|c|c|c|c|c|}
\hline Definition of SF & $\begin{array}{l}\text { Material } \\
\text { strength } \\
\text { parameter }\end{array}$ & $\begin{array}{l}\text { Material } \\
\text { constant } \\
\text { parameter }\end{array}$ & $\begin{array}{l}\text { Lamination } \\
\text { parameter }\end{array}$ & $\begin{array}{l}\text { Dimensional } \\
\text { parameter }\end{array}$ & $\begin{array}{l}\text { Load } \\
\text { parameter }\end{array}$ & $\begin{array}{c}\text { Total number } \\
\text { of random } \\
\text { variables }\end{array}$ \\
\hline Type. I SF1,., & 6 & 4 & 1 & 0 & 0 & 11 \\
Type. II SF2,., & 6 & 4 & 1 & 3 & 3 & 17 \\
Type. II SF3,., & 6 & 4 & 1 & 3 & 3 & 17 \\
\hline
\end{tabular}


Table 2 Characteristic properties of random variables.

(T300/5208)

\begin{tabular}{|c|c|c|c|}
\hline Parameter & Expectation & $\begin{array}{l}\text { Standard } \\
\text { deviation }\end{array}$ & CV \\
\hline$\sigma_{L S}$ & $1: S(x) \quad\left(M P_{a}\right)$ & 150 & 0.10 \\
\hline$\sigma_{\text {uc }}$ & 1500 (MPa) & 180 & 0.12 \\
\hline$\sigma_{r .1}$ & $40 \quad\left(M P_{A}\right)$ & 4.40 & 0.11 \\
\hline$\sigma_{1, c}$ & 246 (MPa) & 19.68 & 0.08 \\
\hline$\sigma_{\Delta r}$ & 68 (MPa) & 4.08 & 0.06 \\
\hline$k_{11}$ & -0.5 & 0.005 & 0.01 \\
\hline$E_{x}$ & 181000 (MPa) & 9050 & 0.05 \\
\hline$E_{y}$ & $10300 \quad(\mathrm{MPa})$ & 515 & 0.05 \\
\hline$E$, & 7170 (MPa) & 358.5 & 0.05 \\
\hline$v_{x}$ & 0.28 (MPa) & 0.0028 & 0.01 \\
\hline$B$ & $30(\mathrm{~mm})$ & 1.5 & 0.05 \\
\hline$L$ & $150 \quad(\mathrm{mn})$ & 7.5 & 0.05 \\
\hline $\boldsymbol{H}$ & $2(m m)$ & 0.1 & 0.05 \\
\hline$P_{1}$ & $90000 \quad(\mathrm{~N})$ & 4500 & 0.05 \\
\hline$P_{2}$ & $12000 \quad(N)$ & 600 & 0.05 \\
\hline$P_{6}$ & 4080 (N) & 20.1 & 0.05 \\
\hline
\end{tabular}

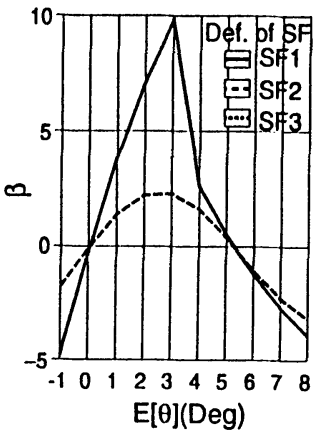

Figure 3 Relation between $\beta$ and $\mathrm{E}[\theta]$ in correspondence to the definition of SF.

stochastic features of parameters and design variables for T300/5208, whose distribution types are respectively

$$
\begin{gathered}
\sigma_{L, t}, \sigma_{L, c}, \sigma_{T, t}, \sigma_{T, c}, \sigma_{L T} \rightarrow \text { Log-normal distribution, } \\
k_{12}, E_{x}, E_{y}, E_{s}, v_{x}, B, L, H \rightarrow \text { Normal distribution, } \\
P_{1}, P_{2}, P_{6} \rightarrow \text { Weibull distribution (2 parameters). }
\end{gathered}
$$

Figure 3 shows one of the typical differences of the value of safety indices on some value of expectation of lamination angles, where each safety factor are decided that

$$
\mathrm{SF}_{(.)}=1.790, \mathrm{SF}_{(.)}=1.810, \mathrm{SF}_{(.)}=1.346
$$

as the nominal mean stresses are equal each other on principle axis by Eq.(21). Despite of the same behavior of in-plane strain for any lamination angles, it is seen that there are great differences between the results among $\mathrm{SF}_{(.)}, \mathrm{SF}_{(.)}$, and $\mathrm{SF}_{(.)}$. Especially the distinctions become to be remarkable around the maximum point of safety index on lamination angle. However the results by $\mathrm{SF}_{(.)}$are same to those by $\mathrm{SF}_{(.)}$whose number of random variables are equal to $\mathrm{SF}({ })$.

For the consideration of this fact, the sensitivity study on random parameters which construct the limit state function was performed. Figure 4 shows the physical and stochastic sensitivities for the expectation degree of the lamination angle. In the figures it is clarified that the load factors such as $\mathrm{P} 1, \mathrm{P} 2, \mathrm{P} 3$ and the dimensional factors such as $\mathrm{B}, \mathrm{L}, \mathrm{H}$ have some 
quantities of physical and stochastic sensitivities as well as strength factors. Nevertheless the modulus factors have little sensitivities. Since the definition of Type I safety factor by Eq.(18) does not include these factors, it is suggested that such a differences of final measure of safety indices arises from the effect of sensitivities of load and dimensional factors.

These evaluation results which show the discrepancies of the value of safety indices on the definition of safety factors are in correspondence with the mean values and coefficient of deviations shown in Table 2. For some kinds of combination of the coefficients of variation of random parameters such as load and strength factors, the effectiveness of the lack of dimensional invariance are examined as described in Figure 5. In the figure, it is seen that the differences by the definition of safety factor become to be remarkable in proportion to the increases of the value of coefficient of variation of load factors.

\section{CONCLUDING REMARKS}

In the practical materials design of composites materials, several definitions are essentially derived because of the intrinsic design specification. However it is verified in this study that
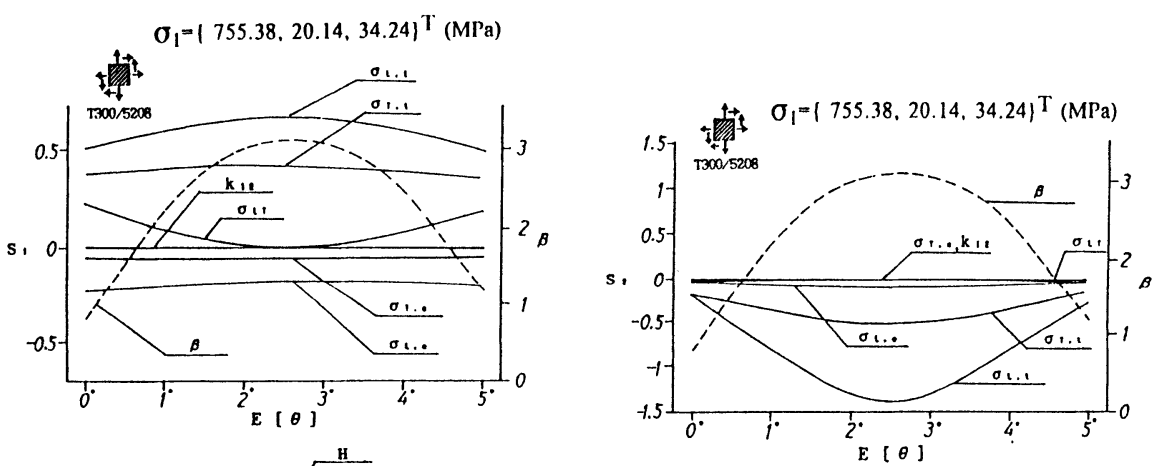

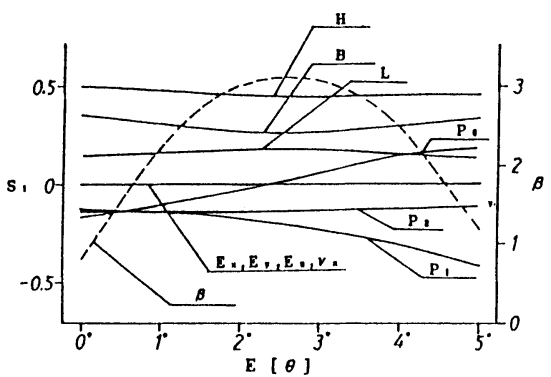

(a) Physical sensitivities

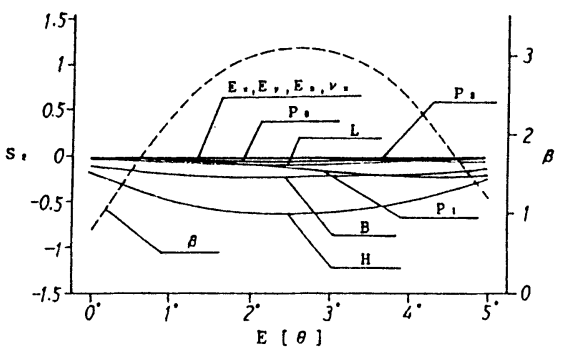

(b) Stochastic sensitivities

Figure 4 Physical and Stochastic sensitivities of random parameters under off axial load condition. 

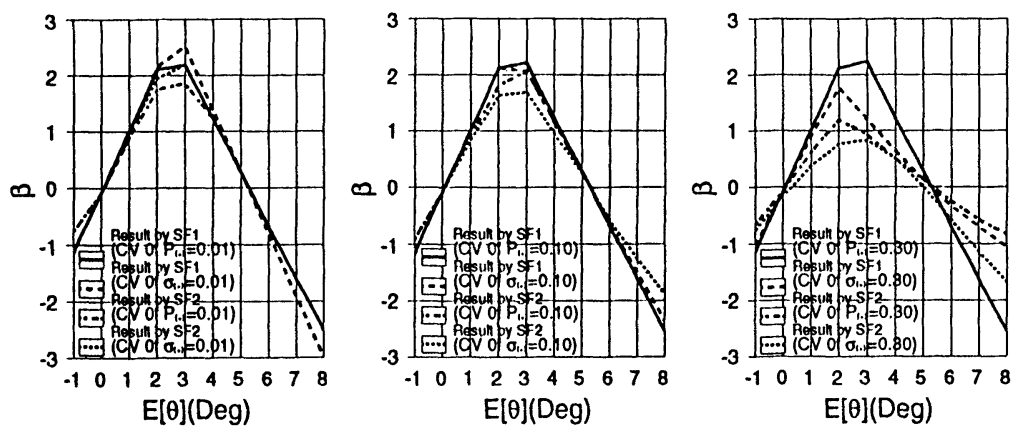

Figure 5 Relation between $\beta$ and $\mathrm{E}[\theta]$ to the combination of $\mathrm{CV}$ of load and strength factors.

some differences of the real safety measures considering parameter uncertainties become to be remarkable in dependence how to select the nominal safety factors in design processes. This discrepancy arises from the lack of dimensional invariability. From the previous experiences of the former study[6], there are little discrepancy of the safety measures in the treatment on inplane stress and strain based limit state functions, though the former has small number of random variables than the latter. The lack of dimensional invariability was negligible problem in the modeling process if the sensitivities of random parameters are small. On the other hand, it should be noted that the lack of dimensional invariability on the design process of stochastic materials design of selecting the nominal safety factor yields great discrepancy of final safety index because of the sensitivities of load and dimensional random parameters. Thus one must be taken into account the differences by the selection of nominal safety factor which arises from the lack of dimensional in variabilities and keep equivalent safety margin whenever any definition of safety factor is specified in the practical materials design of composite materials.

\section{ACKNOWLEDGMENTS}

This work was partly supported by the Science Research Fund of the Ministry Education, Science and Culture of Japan, and the Science Research Promotion Fund of Japan Private School Promotion Foundation. The author thanks to Mr. Fukuzumi (Showa Rekisei Co. Ltd.) for his cooperation to this work.

\section{REFERENCES}

1. Tsai, S. W., Composite Design (4th ed.), THINCK COMPOSITES, 1988.

2. Cornell, C. A., ACI J. 66-12 (1969) 974.

3. Hasofer, A. M., and Lind N., Proc. ASCE, J. Engng. Mech.Div., 100 (1974) 111.

4. Hochenbichler, M., Gollwitzer, S., Kruse, K. and Rackwitz, R., Structural Safety, 4 (1987) 267.

5. Tsai, S. W. and Wu E. M., J. Compos. Mater. 5 (1971) 58.

6. Nakayasu, H., Probabilistic Structural Mechanics: Advances in Structural Reliability Methods (Spanos,P.D. and Wu, Y.-T.Eds.),Springer-Verlag, Berlin Heidelberg, 1994, 392. 